ARTICLE

\title{
A nitrogen-doped nanotube molecule with atom vacancy defects
}

\author{
Koki Ikemoto (1) 1,2, Seungmin Yang (1) 1, Hisashi Naito (1) ${ }^{3}$, Motoko Kotani ${ }^{4,5}$, Sota Sato (1) ${ }^{1,2} \&$ \\ Hiroyuki Isobe (iD) $1,2 \otimes$
}

Nitrogen-doped carbon nanotubes have attracted attention in various fields, but lack of congeners with discrete molecular structures has hampered developments based on indepth, chemical understandings. In this study, a nanotube molecule doped periodically with multiple nitrogen atoms has been synthesized by combining eight 2,4,6-trisubstituted pyridine units with thirty-two 1,3,5-trisubstituted benzene units. A synthetic strategy involving geodesic phenine frameworks is sufficiently versatile to tolerate pyridine units without requiring synthetic detours. Crystallographic analyses adopting aspherical multipole atom models reveal the presence of axially rotated structures as a minor disordered structure, which also provides detailed molecular and electronic structures. The nitrogen atoms on the nanotube serve as chemically distinct sites covered with negatively charged surfaces, and they increase the chance of electron injections by lowering the energy levels of the unoccupied orbitals that should serve as electron acceptors.

\footnotetext{
${ }^{1}$ Department of Chemistry, The University of Tokyo, Hongo, Tokyo 113-0033, Japan. ${ }^{2}$ JST, ERATO, Isobe Degenerate $\pi$-Integration Project, Hongo, Tokyo 113-0033, Japan. ${ }^{3}$ Graduate School of Mathematics, Nagoya University, Nagoya 464-8602, Japan. ${ }^{4}$ Advanced Institute for Materials Research, Tohoku University, Sendai 980-8577, Japan. ${ }^{5}$ Mathematical Institute, Tohoku University, Sendai 980-8578, Japan. ${ }^{凶}$ email: isobe@chem.s.u-tokyo.ac.jp
} 
T he uniqueness of nanoscale graphitic networks is being exploited, and, in particular, nanocarbons containing noncarbon elements attract attention. Nitrogen-doped carbon nanotubes were the first examples of doped nanocarbons ${ }^{1-3}$, and modulations of electronic structures were demonstrated ${ }^{4-6}$. Although donor-type contributions of nitrogen dopants have been taken for granted, due to preceding concepts of doped ntype inorganic semiconductors 7 , effects of nitrogen-doped nanotubes are still controversial ${ }^{4-6}$ because of the lack of sufficient information about atomic-level and electronic structures ${ }^{8}$. Here we show the bottom-up synthesis and high-precision structures of a nitrogen-doped nanotube molecule with a discrete, rigid structure. Taking advantage of a versatile synthetic strategy $^{9,10}$, we replace eight methine $(\mathrm{CH})$ groups of a $(12,12)$ phenine nanotube $(\mathrm{pNT})$ molecule $^{11}$ with eight nitrogen atoms and eight atom vacancy sites (Fig. 1). Detailed molecular and electronic structures are disclosed to reveal unforeseen effects of nitrogen doping.

\section{Results}

Synthesis. By replacing the phenine unit of pNT with 2,4,6-trisubstituted pyridine, i.e., a nitrogen-doped phenine congener, we designed nitrogen-doped pNT molecule $(\mathrm{NpNT})^{11}$. A few minor modifications were made in the synthesis, which resulted in a slightly improved overall yield for the nitrogen-doped variant (Fig. 2). Thus, the starting material was changed from dibromobenzene to 1-bromo-3-chlorobenzene, which allowed the coupling reaction with 2,6-dibromopyridine (4) via silylation and borylation, and a resultant terphenylene congener (5) was coupled by a Yamamoto-type coupling reaction to afford a nitrogendoped congener of [6]cyclo-meta-phenylene ([6]CMP) (6) with an improved yield. The nitrogen-doped [6]CMP congener was then cyclized to a flexible cyclic GPF (8) through borylation, Pt-mediated macrocyclization, ligand exchange and reductive elimination ${ }^{12}$. The final three steps of iododesilylation, Suzuki-Miyaura coupling and Yamamoto-type coupling completed the nanometre-sized cylinder molecule, $(12,12)$-NpNT. A minor modification of the catalyst for Suzuki-Miyaura coupling was also made, and the overall yield of $(12,12)$-NpNT was improved to $1.4 \%$ for the 10 -step synthesis from the value of $0.7 \%$ that was recorded for the 9-step synthesis of $(12,12)-\mathrm{pNT}^{11}$. The structure of $(12,12)$-NpNT was first established by spectroscopy. The ${ }^{1} \mathrm{H}$ NMR spectrum showed seven aromatic singlet resonances (Fig. 2), which showed the loss of one methine resonance

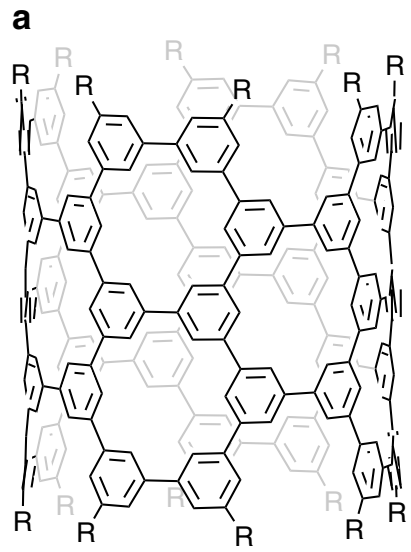

$(12,12)-p N T, \mathrm{C}_{304} \mathrm{H}_{264}$ $t_{\mathrm{f}}=7.0, F_{\mathrm{b}}=67 \%, F_{\mathrm{a}}=67 \%$

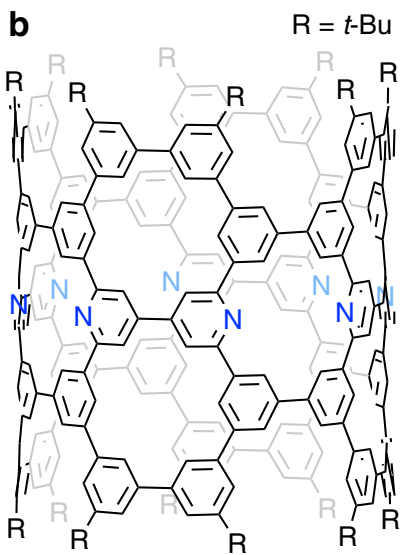

$(12,12)-\mathrm{NpNT}, \mathrm{C}_{296} \mathrm{H}_{256} \mathrm{~N}_{8}$ $t_{\mathrm{f}}=7.0, F_{\mathrm{b}}=53 \%, F_{\mathrm{a}}=64 \%$
Fig. 1 Phenine nanotube molecules. a (12,12)-pNT. b Nitrogen-doped $(12,12)-N p N T$. relative to the eight aromatic resonances of $(12,12)-\mathrm{pNT}^{11}$. The chemical composition of $\mathrm{C}_{296} \mathrm{H}_{256} \mathrm{~N}_{8}$ was confirmed by a MALDI-TOF mass spectrum showing the presence of an ionized species with $m / z=3923[\mathrm{M}+\mathrm{H}]^{+}$. In the chemical composition, the nitrogen atoms occupy $2.6 \%$ of non-hydrogen atoms (8/304), which is close to the nitrogen contents often reported for nitrogen-doped carbon nanotubes $(2-5 \%)^{2,4}$. Representative geometric descriptions of the molecule are summarized as follows. The (12,12)-NpNT molecule possesses a graphitic lattice of $(12,12)$-carbon nanotube with a length index of $t_{\mathrm{f}}=7.0$ (ref. ${ }^{13}$ ). The structural defects comprise both replacements and depletions of atoms and bonds (Supplementary Fig. 1), which can be quantitatively described by geometric measures of bond-filling and atom-filling indices of $F_{\mathrm{b}}=53 \%$ and $F_{\mathrm{a}}=64 \%$. By using an oblique coordinate system of carbon nanotubes (see the Supplementary Methods for details $)^{13,14}$, we can further identify and describe the positions of nitrogen atoms as $(5,-2),(8,1),(11 / 3$, $-10 / 3),(11,4),(14,7),(20 / 3,-1 / 3),(29 / 3,8 / 3)$, and $(38 / 3,17 / 3)$ (Supplementary Fig. 2).

Crystallography. For the elucidation of precise molecular structures, elaborate X-ray diffraction analyses were necessary because of unexpected, disordered structures unique to the cylindrical shape. A single crystal of $(12,12)-N p N T$ was obtained from a 1,2dichloroethane solution by diffusing 2-propanol vapour at $25^{\circ} \mathrm{C}$. With 22,961 unique reflections from 299,140 total reflections (multiplicity of observations $=13$ ) from a single crystal, we first solved the structure by a conventional analytical method adopting spherical, independent atom models (IAM) ${ }^{15}$. With a few $t$-Bu conformations located as disordered structures, the structure converged with a moderate $R$-factor $\left[R\left(F^{2}\right)=0.1267\right.$ on SHELX ${ }^{16}$ and $R(F)=0.1010$ on XD2016 ${ }^{17}$ (Supplementary Fig. 4, stage 0 and stage 1). To define a structure with a higher accuracy, we adopted multipole aspherical atom models in the Hansen-Coppens formalism ${ }^{18}$ by using a transferrable aspherical atom model (TAAM) ${ }^{19}$ with parameters from the University at Buffalo pseudoatom databank (UBDB) ${ }^{20}$ (Supplementary Fig. 3). Close examinations of the residual electron densities $\left(F_{\mathrm{o}}-F_{\mathrm{c}}\right)$ after the TAAM analysis indicated the presence of a disordered structure that originated from a $45^{\circ}$ axial rotation of the nanometre-sized cylinder (Supplementary Fig. 4; stage 2). Such rotational disorders were not found with a previous example of hydrocarbon $\mathrm{pNT}^{11}$. With this anomalous rotational disorder included as a minor structure (occupancy $=12 \%$ ), the molecular structures were solved with a higher accuracy (Fig. 3a): the $R(F)$ values reached 0.0855 with IAM/XD2016 analysis (Supplementary Fig. 4, stage 3), which was further improved to $R(F)=0.0802$ with TAAM/XD2016 analysis with multipole aspherical atom models (Supplementary Fig. 4 , stage 4 ). The $F_{\mathrm{o}}-F_{\mathrm{c}}$ maps confirmed the decrease in the residual electron densities (Supplementary Fig. 4), and the deformation maps of $F_{1}-F_{2}$ confirmed the deformed densities of the major structure. The two axial rotational disorders were made possible by the cylindrical shape with uniform diameters $(1.6 \mathrm{~nm}$; Supplementary Fig. 5). The nanometre-sized cylindrical structure is shown for the major disorder (occupancy $=88 \%$ ) in Fig. $3 \mathrm{~b}$. The introduction of eight nitrogen atoms did not affect the overall molecular structures, and negligible differences between $(12,12)$-NpNT and $(12,12)$ pNT were noted with the $\pi$-orbital axis vector (POAV) $\theta_{\mathrm{p}}$ and dihedral angles ${ }^{21}$ (Supplementary Fig. 6).

Electronic structures. The effects of the nitrogen dopants were determined from the fine molecular structures. As observed from the crystal structure of 4,4'-bipyridine, the lengths of $\mathrm{C}-\mathrm{N}$ bonds in the aromatic ring are shorter than the lengths of the $\mathrm{C}-\mathrm{C}$ 
a
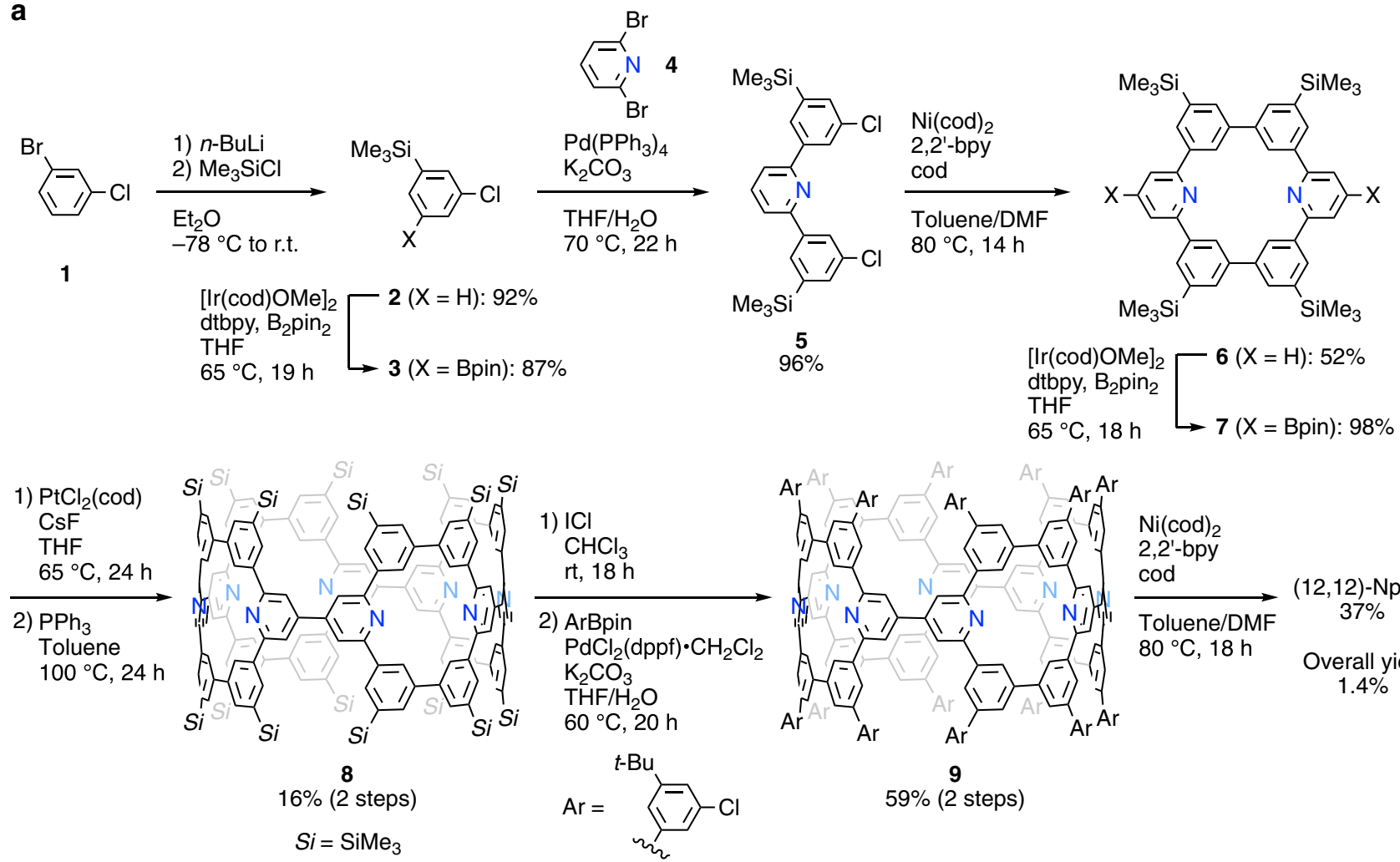

$\mathrm{Ni}(\operatorname{cod})_{2}$

2,2'-bpy

cod

$(12,12)-\mathrm{NpNT}$

$\underset{\text { Toluene/DMF }}{\longrightarrow} \quad 37 \%$

$80^{\circ} \mathrm{C}, 18 \mathrm{~h}$

Overall yield $1.4 \%$

b

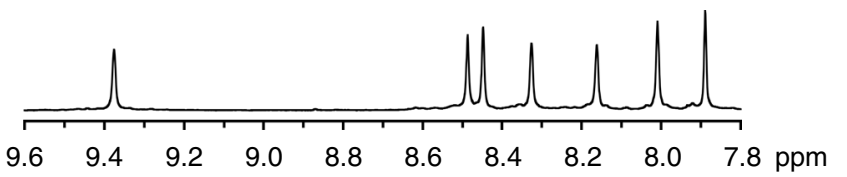

C

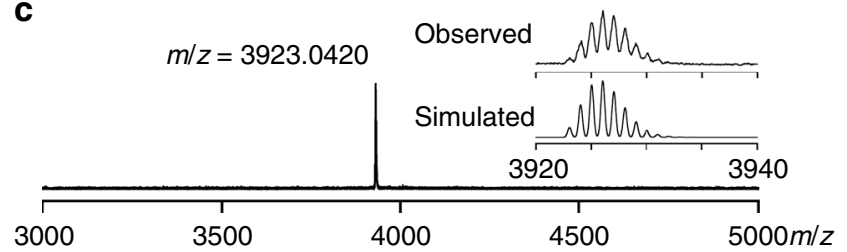

Fig. 2 Synthesis of (12,12)-NpNT. a Synthesis. b A proton NMR spectrum in $\mathrm{CDCl}_{3}$ at $298 \mathrm{~K}$. c A MALDI-TOF mass spectrum (dithranol matrix, positive).

bonds (1.34 vs. $1.39 \AA$ ) (Supplementary Fig. 7). Unlike flexible cycloarylenes with fluctuating structures 22,23 , (12,12)-NpNT possessed a rigid molecular structure, which allowed for unequivocal assignments of the nitrogen atoms. The crystallographic analyses of $(12,12)-\mathrm{NpNT}$ thus allocated the shorter bonds of $1.33 \AA$ at the expected positions to connect the nitrogen atoms (Supplementary Fig. 7). The effects of nitrogen atoms and their bonding on the electron density were further clarified by charge-density analysis after TAAM/XD2016 refinements ${ }^{18,19}$. The deformation map showing the electron density deformed by chemical bonds thus revealed the lone pair densities on the nitrogen atoms (Fig. 3c). An electrostatic potential map also revealed the charge distributions and located the red areas of higher electron densities around the nitrogen atoms, characterizing the nitrogen sites as chemically distinct areas with an abundance of electrons (Fig. 3d).

Optical properties. The effects of nitrogen doping were also observed from the optical properties. As shown in Fig. 4a, the UV absorption spectrum of $(12,12)-\mathrm{NpNT}$ was redshifted from that of non-doped $(12,12)$-pNT $\left(\lambda_{\text {edge }}=345 \mathrm{~nm}\right)^{11}$, with the edge absorption appearing at $378 \mathrm{~nm}$ (optical gap $=3.28 \mathrm{eV}$ ). Fluorescence was also observed with $(12,12)-\mathrm{NpNT}$ with a quantum yield of $16 \%$. The density functional theory (DFT) calculations of $(12,12)-\mathrm{NpNT}$ also confirmed the narrowed gap between the highest occupied molecular orbital (HOMO) and the lowest unoccupied molecular orbital (LUMO) (3.07 eV) with highly degenerate orbitals (Fig. 4b). However, the lone pairs of nitrogen did not contribute to the narrowing of the gap, with HOMO-7 to HOMO-10 (-3.55 eV) located below HOMO to HOMO-6 among the conjugated $\pi$-orbitals $(-3.48 \mathrm{eV}$ ) (Fig. $4 \mathrm{~b}$ and Supplementary Fig. 9). In contrast, the nitrogen-related $\pi^{*}$-orbitals were inserted on the unoccupied orbital side as LUMO to LUMO+3 at -0.41 to $-0.38 \mathrm{eV}$, which narrowed the HOMO-LUMO gap of the cylindrical molecule. The effects of nitrogen atoms over the electronic structures of NpNT were unexpected from $\mathrm{HOMO} /$ LUMO-stabilizations found with $\mathrm{N}$-doped acenes ${ }^{24}$.

The optical properties were modulated under acidic conditions. When we added an excess amount of trifluoroacetic acid, the absorption and fluorescence spectra were broadened (Fig. 4d). Moreover, when photoluminescence quantum yields were measured, the quantum yield dropped from $16 \%$ to $8 \%$ under the acidic conditions. We believe that the spectral changes can be ascribed, most likely, to the protonation at the pyridinic nitrogen atoms of NpNT under acidic conditions.

\section{Discussion}

A nitrogen-doped congener of phenine nanotubes was synthesized, which also allowed us to periodically embed atom vacancy defects into a nanotube molecule. Crystallographic analyses adopting aspherical atomic models revealed the presence of unique disorders with axially rotated molecular structures, which 
a

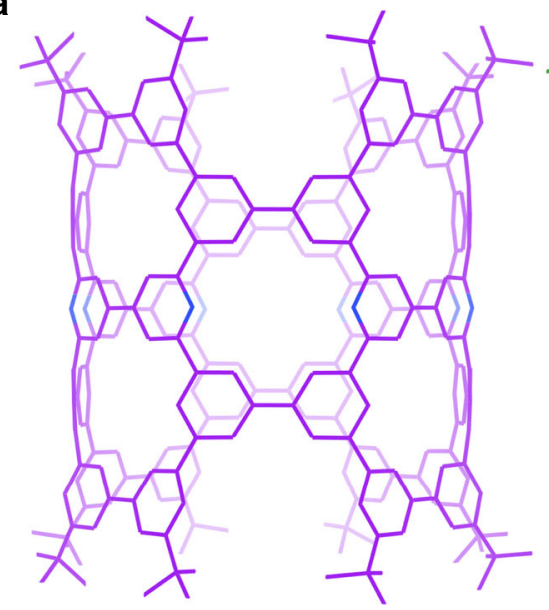

Occupancy $88 \%$

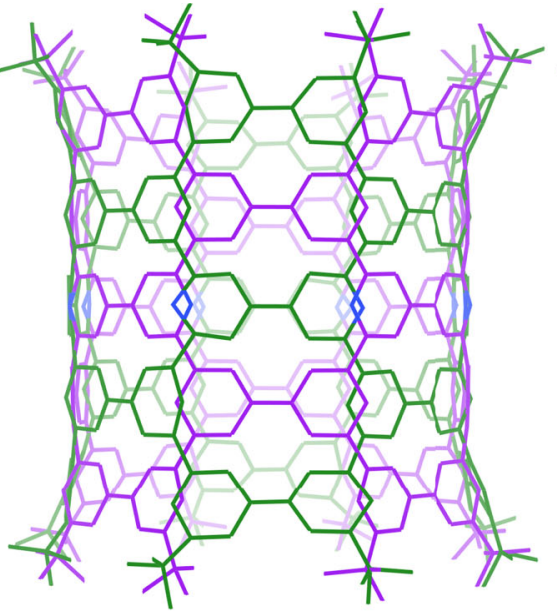

Overlay of $45^{\circ}$ axial rotational disorders

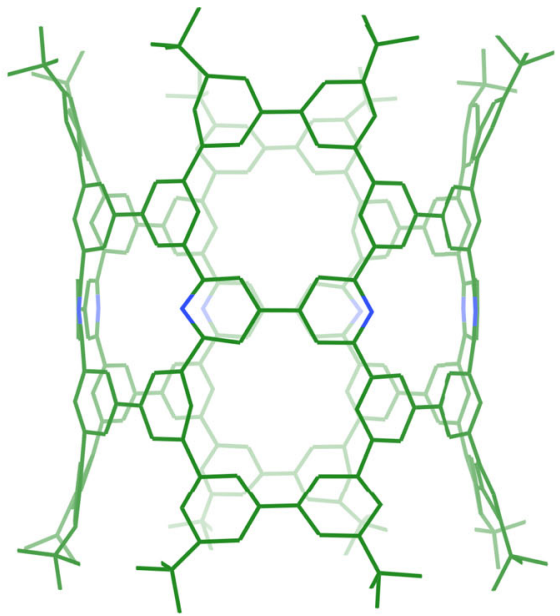

Occupancy $12 \%$ b

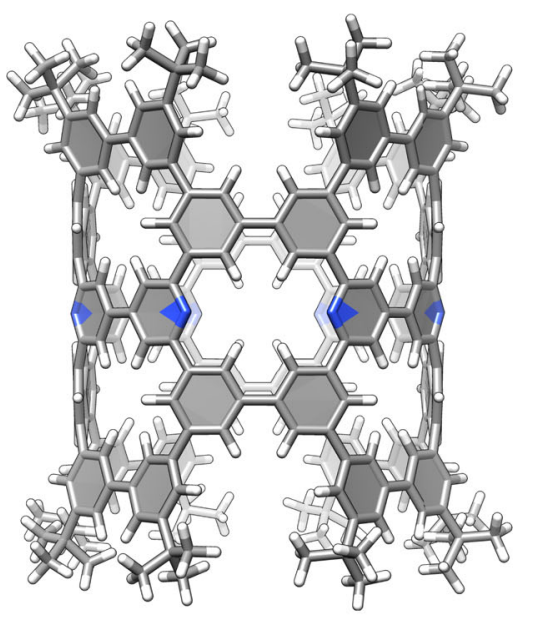

C

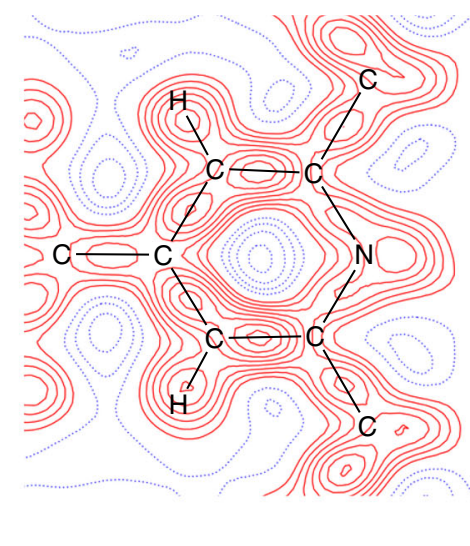

d

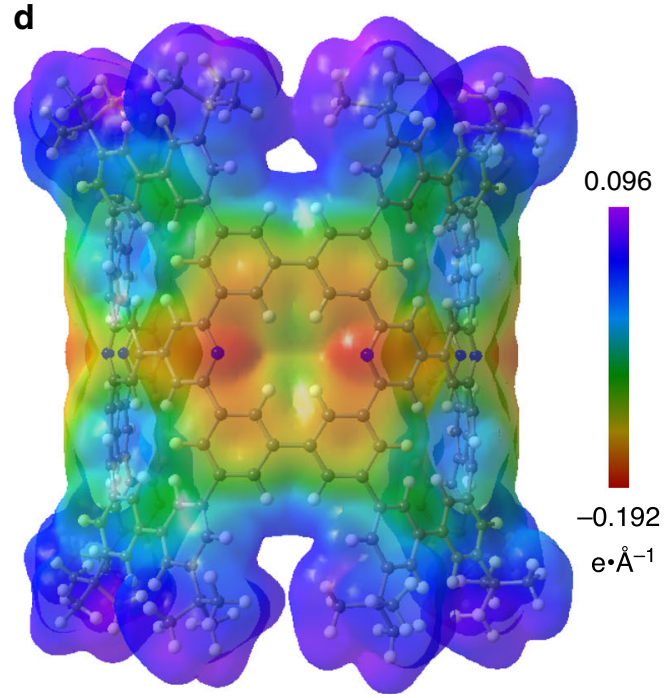

Fig. 3 Crystal structures of (12,12)-NpNT. a Molecular structures of two disordered structures. b Molecular structure (occupancy $=88 \%$ ). c Deformation map (contour interval: 0.02 e $\AA^{-3}$, positive: red, negative: blue). Note that, because of the lower occupancy of $12 \%$, negligible densities of the minor disordered structure were found in the electron and deformation density map, which also resulted in distortions of the assigned structures. See also Fig. $3 a$ and Supplementary Fig. 5. d Electrostatic potential map mapped on the 0.0067 e $\AA^{-3}$ isosurface of the electron density.

should similarly exist in bundles of carbon nanotubes. The aspherical atom models also allowed charge density analyses, showing the subtle yet apparent effects of nitrogen atoms on the structures. Interestingly, the nitrogen lone pairs created chemically distinct, basic sites on the molecule but did not affect the HOMO level. In contrast, the nitrogen-related $\pi^{*}$-orbitals lowered the LUMO level, which should be favourable in n-type organic semiconductors by accepting negative charge carriers. Thus, unlike the inorganic semiconductors where lone pairs donate charge carriers in the conduction bands, the nitrogendoped nanotubes tend to be n-type organic semiconductors where lowered unoccupied orbitals facilitate electron injections from the electrodes. Thus, this study revealed unique characteristics of so-called pyridinic nitrogen atoms doped on graphitic networks ${ }^{4-6}$. This study also demonstrated that the structure-property relationships of nanocarbon molecules, particularly those with rigid discrete structures, can deepen our understanding of the effects of heteroatom doping ${ }^{25}$. Taking advantage of the versatile synthetic approach of phenine frameworks, we will explore other variants of heteroatom dopants with various locations and structures of dopants in the near future.

\section{Methods}

Synthesis. The nitrogen-doped $(12,12)$-NpNT was synthesized by the procedure detailed in the Supplementary Methods. All the data necessary for the identification are also provided.

Location of defects. Locations of defects were defined by using an oblique coordinate system of carbon nanotubes ${ }^{14}$. Details such as the location of the origin are described in the Supplementary Methods. The defect locations can also be defined by using a web-based applet at https://physorg.chem.s.u-tokyo.ac.jp/applet/defect/.

Crystallography. A single crystal (ca. $0.04 \times 0.03 \times 0.02 \mathrm{~mm}^{3}$ ) suitable for the crystallographic analysis was obtained by slowly diffusing 2-propanol vapour into a solution of $(12,12)-\mathrm{NpNT}$ in 1,2-dichloroethane at $25^{\circ} \mathrm{C}$. A single crystal was mounted on a thin polymer tip with cryoprotectant oil and frozen at $-173^{\circ} \mathrm{C}$ via flash-cooling. The diffraction analysis with a synchrotron X-ray source was conducted at $-173^{\circ} \mathrm{C}$ at the beamline BL26B1 (SPring-8), which was collimated for an increased brightness with a capillary lens (Hamamatsu, J12432-01). The beamline for macromolecular crystallography was equipped with a single-axis goniometer (KOHZU, QKSU-SDD) with an additional house-build compact $\kappa$ goniometer head for the crystal tilt. Diffraction data were collected by a hybrid photon counting detector (Dectris EIGER X 4M) comprising $2070 \times 2167$ pixels up to $\sin \theta \lambda^{-1}=0.60 \AA^{-1}$ resolution $^{26}$. The first set of diffractions were collected with an oscillation range of $0.1^{\circ}$ (exposure time $=0.3 \mathrm{~s}$ ) for a $\omega$ range of $360^{\circ}$. Because of an insufficient level of the completeness $(94.6 \%)$, a second set of $360^{\circ}$-diffractions were collected after tilting the crystal by $30^{\circ}$. Two datasets were merged with the XDS 

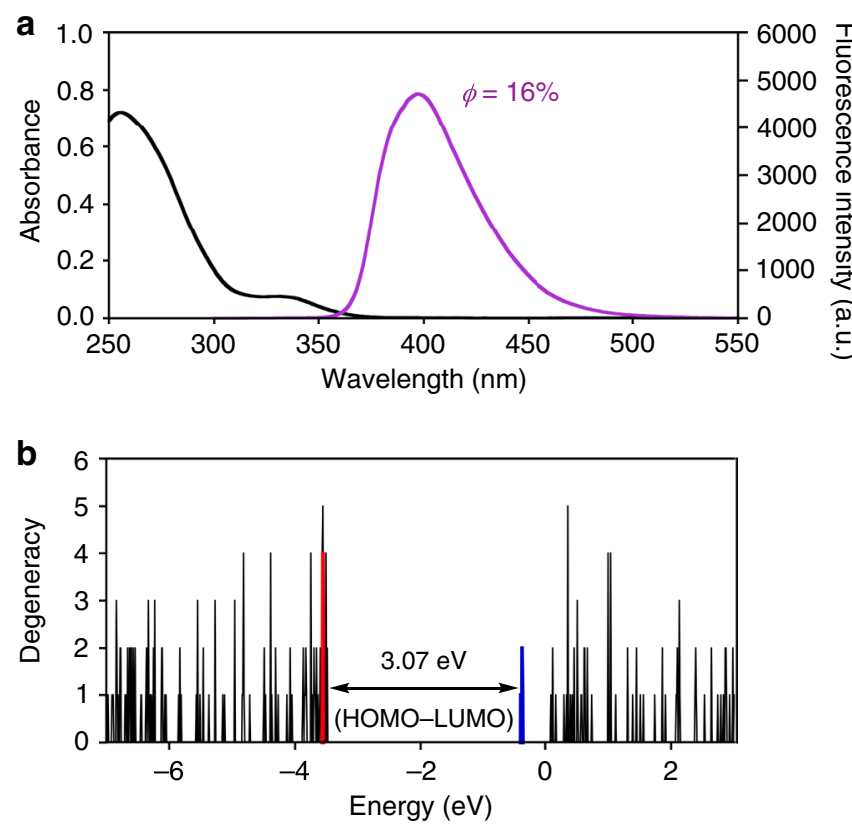

C
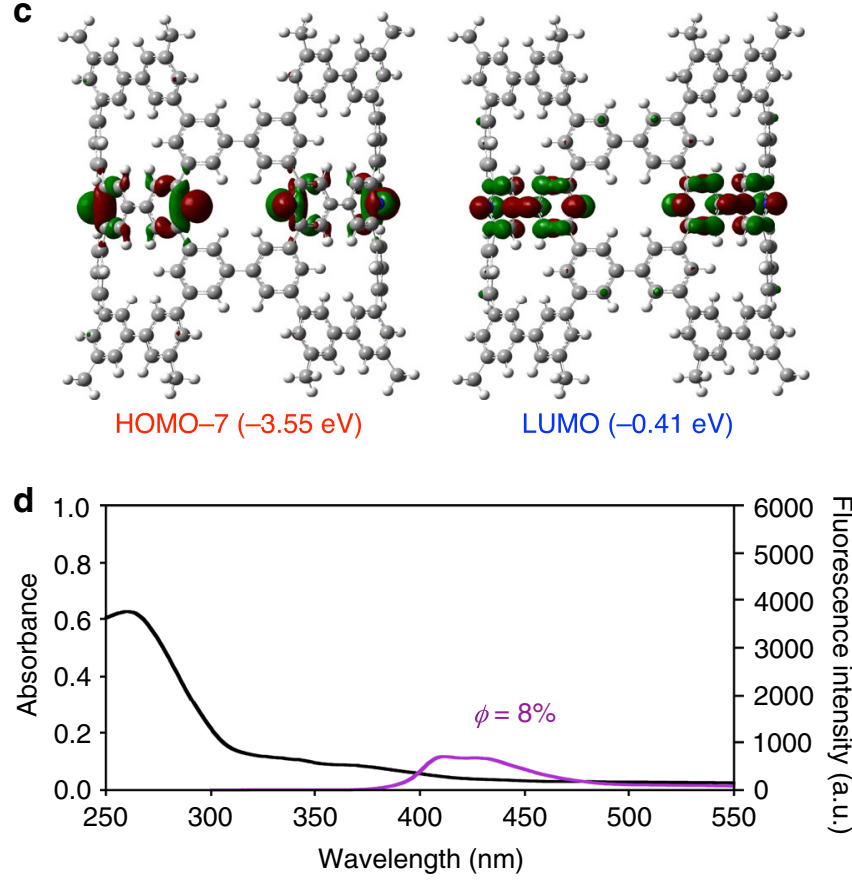

Fig. 4 Representative properties of (12,12)-NpNT. a Absorption and fluorescence spectra of $(12,12)-\mathrm{NpNT}\left(1.2 \times 10^{-6} \mathrm{M}\right.$ in $\left.\mathrm{CH}_{2} \mathrm{Cl}_{2}, 25^{\circ} \mathrm{C}\right)$. b Histogram sorting 213 orbitals of $(12,12)-N p N T$ by energy on the basis of the DFT calculations. Orbitals originating from pyridine units are coloured in red (occupied) and blue (unoccupied). See Supplementary Fig. 9 for details. c Representative Kohn-Sham orbitals. d Absorption and fluorescence spectra of $(12,12)-\mathrm{NpNT}\left(1.2 \times 10^{-6} \mathrm{M}\right.$ in $\left.\mathrm{CH}_{2} \mathrm{Cl}_{2}, 25^{\circ} \mathrm{C}\right)$ in the presence of trifluoroacetic acid $\left(7.4 \times 10^{-3} \mathrm{M}\right)$.

programme to afford 299,140 total reflections with 22,961 unique reflections (multiplicity of observations $=13)^{27}$. After solving the initial structure by a direct method by using SHELXT software programme $\mathrm{e}^{28}$, the final structure was obtained through trial-and-error processes adopting IAM/SHELXL ${ }^{16}$, IAM/XD2016 and TAAM/XD2016 refinement protocols ${ }^{17}$. In short, the first candidate structure with $t$-Bu disorders was obtained by IAM/SHELXL and IAM/XD2016 refinements but was partly refuted by TAAM/XD2016 refinements with inferior $R$ values. The residual electron densities after the IAM/XD2016 refinements indicated the presence of an unexpected disordered cylinder that was located at axially rotated orientations. By taking account of the minor rotated disorder, we then finalized the structures through IAM/SHELXL, IAM/XD2016 and TAAM/XD2016 refinements. Further details of the refinements and analyses are described in the Supplementary Methods. In addition to our final cif file (CCDC 1966650), we also deposited a preliminary cif file at the stage 0 as CCDC 1984802. The res and HKL data embedded in this file can allow readers to follow the present analytical procedures from the raw data.

DFT calculations. The DFT calculations were performed by the same method used for $(12,12)-\mathrm{pNT}^{11}$. The geometry optimizations were thus performed at the PBEPBE/STO-3G level of theory 29,30 by using Gaussian 16 programme suite ${ }^{31}$.

Optical properties. A solution of $(12,12)-\mathrm{NpNT}$ was prepared in $\mathrm{CH}_{2} \mathrm{Cl}_{2}$ at $1.2 \times$ $10^{-6} \mathrm{M}$. The absorption and fluorescent spectra were recorded at $25^{\circ} \mathrm{C}$ on V-670 (JASCO) and FP-8500 (JASCO; excitation $=255 \mathrm{~nm}$ ) spectrometers, and the photoluminescence quantum yield was determined by C9920-02G spectrometer (Hamamatsu; excitation $=255 \mathrm{~nm}$ ). Fluorescence lifetime was determined as $3.3 \mathrm{~ns}$ on Quantaurus-QY C11347 (Hamamatsu). The same spectra were also recorded after the addition of trifluoroacetic acid $\left(7.4 \times 10^{-3} \mathrm{M}\right)$.

\section{Data availability}

Synthetic and experimental procedures, as well as crystallographic, spectroscopic and computational data are provided in the Supplementary Information. Crystallographic data for the structures reported in this Article have been deposited at the Cambridge Crystallographic Data Centre, under deposition numbers CCDC 1966650, CCDC 1966651 and CCDC 1984802. Copies of the data can be obtained free of charge via www. ccdc.cam.ac.uk/data_request/cif. For the designation of defect locations, we provide a web-based applet that can be used free of charge at https://physorg.chem.s.u-tokyo.ac.jp/ applet/defect/.

Received: 3 December 2019; Accepted: 18 March 2020; Published online: 14 April 2020

\section{References}

1. Yi, J.-Y. \& Bernholc, J. Atomic structure and doping of microtubules. Phys. Rev. B 47, 1708-1711 (1993).

2. Stephan, O. et al. Doping graphitic and carbon nanotube structures with boron and nitrogen. Science 266, 1683-1685 (1994).

3. Terrones, M. et al. Controlled production of aligned-nanotube bundles. Nature 388, 52-55 (1997).

4. Ayala, P., Arenal, R., Rümmeli, M., Rubio, A. \& Pichler, T. The doping of carbon nanotubes with nitrogen and their potential applications. Carbon $\mathbf{4 8}$, 575-586 (2010)

5. Cirić-Marjanović, G., Pašti, I. \& Mentus, S. One-dimensional nitrogencontaining carbon nanostructures. Prog. Mater. Sci. 69, 61-182 (2015)

6. Inagaki, M., Toyoda, M., Soneda, Y. \& Morishita, T. Nitrogen-doped carbon materials. Carbon 132, 104-140 (2018).

7. Sze, S. M. \& Lee, M. K. Semiconductor Devices: Physics and Technology 3rd edn (Wiley \& Sons, 2012)

8. International Union of Pure and Applied Chemistry. Compendium of Chemical Terminology 2nd edn (eds McNaught, A. D. \& Wilkinson, A.) (Blackwell Scientific Publications, 1997).

9. Ikemoto, K., Kobayashi, R., Sato, S. \& Isobe, H. Synthesis and bowl-in-bowl assembly of a geodesic phenylene bowl. Angew. Chem. Int. Ed. 56, 6511-6514 (2017)

10. Ikemoto, K., Lin, J., Kobayashi, R., Sato, S. \& Isobe, H. Fluctuating carbonaceous networks with a persistent molecular shape: a saddle-shaped geodesic framework of 1,3,5-trisubstituted benzene (phenine). Angew. Chem. Int. Ed. 57, 8555-8559 (2018).

11. Sun, Z. et al. Finite phenine nanotubes with periodic vacancy defects. Science 363, 151-155 (2019).

12. Sun, Z., Mio, T., Ikemoto, K., Sato, S. \& Isobe, H. Synthesis, structures, and assembly of geodesic phenine frameworks with isoreticular networks of $[n]$ cyclo-para-phenylenes. J. Org. Chem. 84, 3500-3507 (2019).

13. Matsuno, T. et al. Geometric measures of finite carbon nanotube molecules: a proposal for length index and filling indexes. Pure Appl. Chem. 86, 489-495 (2014).

14. Saito, R., Dresselhaus, G. \& Dresselhaus, M. S. Physical Properties of Carbon Nanotubes (Imperial College Press, 1998).

15. Doyle, P. A. \& Turner, P. S. Relativistic Hartree-Fock X-ray and electron scattering factors. Acta Crystallogr. A 24, 390-397 (1968).

16. Sheldrick, G. M. A short history of SHELX. Acta Crystallogr. A 64, 112-122 (2008). 
17. Volkov, A. et al. XD2016 - a computer program package for multipole refinement, topological analysis of charge densities and evaluation of intermolecular energies from experimental and theoretical structure factors (Univ. Glasgow, 2016).

18. Hansen, N. K. \& Coppens, P. Testing aspherical atom refinements on smallmolecule data sets. Acta Crystallogr. A 34, 909-921 (1978).

19. Brock, C. P., Dunitz, J. D. \& Hirshfeld, F. L. Transferability of deformation densities among related molecules: atomic multipole parameters from perylene for improved estimation of molecular vibrations in naphthalene and anthracene. Acta Crystallogr. B 47, 789-797 (1991).

20. Dominiak, P. M., Volkov, A., Li, X., Messerschmidt, M. \& Coppens, P. A theoretical databank of transferable aspherical atoms and its application to electrostatic interaction energy calculations of macromolecules. J. Chem. Theory Comput. 3, 232-247 (2007).

21. Haddon, R. C. Comment on the relationship of the pyramidalization angle at a conjugated carbon atom to the $\sigma$ bond angles. J. Phys. Chem. 105, 4164-4165 (2001).

22. Matsui, K., Segawa, Y. \& Itami, K. Synthesis and properties of cycloparaphenylene-2,5-pyridylidene: a nitrogen-containing carbon nanoring. Org. Lett. 14, 1888-1891 (2012).

23. Darzi, E. R. et al. Synthesis, properties, and design principles of donor-acceptor nanohoops. ACS Cent. Sci. 1, 335-342 (2015).

24. Bunz, U. H. F. The larger linear N-heteroacenes. Acc. Chem. Res. 48, 1676-1686 (2015).

25. Myśliwiec., D., Kondratowicz, M., Lis, T., Chmielewski, P. J. \& Stępień, M. Highly strained nonclassical nanotube end-caps. A single-step solution synthesis from strain-free, non-macrocyclic precursors. J. Am. Chem. Soc. 137, 1643-1649 (2015).

26. Förster, A., Brandstetter, S. \& Schulze-Briese, C. Transforming X-ray detection with hybrid photon counting detectors. Philos. Trans. R. Soc. A 362, 20180241 (2019).

27. Kabsch, K. Automatic processing of rotation diffraction data from crystals of initially unknown symmetry and cell constants. J. Appl. Crystallogr. 26, 795-800 (1993).

28. Sheldrick, G. M. SHELXT-integrated space-group and crystal-structure determination. Acta Crystallogr. A 71, 3-8 (2015).

29. Perdew, J. P., Burke, K. \& Ernzerhof, M. Generalized gradient approximation made simple. Phys. Rev. Lett. 77, 3865-3868 (1996).

30. Hehre, W. J., Stewart, R. F. \& Pople, J. A. Self-consistent molecular-orbital methods. I. Use of Gaussian expansions of Slater-type atomic orbitals. J. Chem. Phys. 51, 2657-2664 (1969).

31. Frisch, M. J. et al. Gaussian 16, Revision B.01 (Gaussian, Inc., 2016).

\section{Acknowledgements}

We thank Professors B. Dittrich (Heinrich-Heine-Universität Düsseldorf) and T. Koritsanszky (Middle Tennessee State University) for their kind advices on TAAM/XD2016 analyses. This study is partly supported by JST ERATO (JPMJER1301) and KAKENHI (17H01033, 17K05772 and 19H02552). We were granted access to the X-ray diffraction instruments in SPring-8 BL26B1 beamline (nos. 2018B2719 and 2019A1181) and KEK Photon Factory (2019G051).

\section{Author contributions}

H.I. launched the project, K.I. and H.I. conceived the design, and K.I. and S.Y. synthesized the target. K.I., H.N., M.K. and H.I. developed defect descriptors. K.I., S.Y., S.S. and H.I. performed the crystallographic studies, and K.I. and S.Y. performed the DFT calculations. All authors analysed and discussed the results, and K.I. and H.I. wrote the manuscript.

\section{Competing interests}

The authors declare no competing interests.

\section{Additional information}

Supplementary information is available for this paper at https://doi.org/10.1038/s41467020-15662-6.

Correspondence and requests for materials should be addressed to H.I.

Peer review information Nature Communications thanks the anonymous reviewer(s) for their contribution to the peer review of this work. Peer reviewer reports are available.

Reprints and permission information is available at http://www.nature.com/reprints

Publisher's note Springer Nature remains neutral with regard to jurisdictional claims in published maps and institutional affiliations.

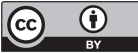

Open Access This article is licensed under a Creative Commons Attribution 4.0 International License, which permits use, sharing, adaptation, distribution and reproduction in any medium or format, as long as you give appropriate credit to the original author(s) and the source, provide a link to the Creative Commons license, and indicate if changes were made. The images or other third party material in this article are included in the article's Creative Commons license, unless indicated otherwise in a credit line to the material. If material is not included in the article's Creative Commons license and your intended use is not permitted by statutory regulation or exceeds the permitted use, you will need to obtain permission directly from the copyright holder. To view a copy of this license, visit http://creativecommons.org/ licenses/by/4.0/

(C) The Author(s) 2020 